\title{
Traumatic Pediatric Atlantoaxial Rotatory Subluxation Successfully Reduced and Discharged from the Emergency Department: A Case Report
}

Merritt D Kinon $^{1 *}$, Rani Nasser ${ }^{1}$, Jonathan P Nakhla1, Reza Yassari', Rupen Desai ${ }^{2}$ and Carlos A Bagley ${ }^{3}$

${ }^{1}$ Montefiore Medical Center/Albert Einstein College of Medicine, Department of Neurosurgery, Bronx, NY, USA

${ }^{2}$ Duke University School of Medicine, Durham, NC, USA

${ }^{3}$ University of Texas Southwestern Medical Center, Department of Neurosurgery, Dallas, TX, USA

\begin{abstract}
The incidence of atlantoaxial rotatory subluxation is fairly uncommon; however it is more common to be encountered in the pediatric population. It is usually seen in children after a retropharyngeal inflammation or after a minor trauma. Children have unique anatomical features such as inherent ligamentous laxity and shallower and more horizontally oriented facet joints that allow greater freedom of motion especially at the C1-2 joint making them prone to atlantoaxial subluxation. If recognized early it can be successfully managed nonsurgically before chronic inflammatory changes affect the ligaments and joint structure of the C1-2 complex. However, it is unclear in the literature if inpatient admission is necessary for treatment. Here we present a case of atlantoaxial rotatory subluxation in a child caused by forcefully throwing a ball that was successfully reduced and subsequently discharge from the pediatric emergency department all in the same visit.
\end{abstract}

Keywords: Cervical spine; Trauma; Atlantoaxial subluxation; Torticollis

\section{Key Messages}

There is no clear criterion for inpatient admission of pediatric atlantoaxial rotatory subluxation. We present a unique case of atlantoaxial rotatory subluxation in a child after the benign act of throwing a ball that was successfully treated and discharged home from the emergency department in the same visit with close outpatient follow up.

\section{Introduction}

In the pediatric population atlantoaxial rotatory subluxation usually presents with acute onset torticollis either after a retropharyngeal inflammation or minor trauma [1-4]. Its occurrence is relatively rare, but swift recognition and treatment especially in the pediatric emergency department is paramount [2,5-9]. The speed of diagnosis, degree of associated subluxation, and involvement of associated ligamentous structures affects treatment. Over time, chronic inflammatory changes in the ligaments and joint structure of the atlantoaxial complex limit more conservative treatment options [1,8]. Diagnosis is achieved through a careful history and physical examination with the help of radiologic evaluation, such as plain radiographs of the cervical spine and computed tomography [8]. Here we report a case of atlantoaxial rotary subluxation in a pediatric patient presenting with torticollis after the benign trauma of throwing a baseball that was successfully reduced in the emergency department with manual manipulation under minor procedural sedation and show that if the patient and family are comfortable and motivated they can be discharged from the emergency department shortly after reduction and managed successfully in a hard cervical collar as an outpatient.

\section{Case History}

A 9-year-old boy with no significant past medical history or recent viral illness was brought to the emergency department by his mother with chief complaint of neck pain and inability to straighten his head after throwing a ball to his grandmother earlier in the afternoon. The family described that the patient developed intense neck pain with his head locked towards the right with inability to turn back to midline after a particularly forceful throw. Upon physical examination, the patient's head was fixed approximately 45 degrees to the right and slightly rotated downward. He was neurologically intact with no sensory or motor deficits. CT of the cervical spine showed an anterior shift of the arch of $\mathrm{C} 1$ of approximately $4.5 \mathrm{~mm}$ as well as rotation of $\mathrm{C} 1$ on $\mathrm{C} 2$ of approximately 40 degrees with the right lateral mass of $\mathrm{C} 1$ displaced $8 \mathrm{~mm}$ anteriorly and the left lateral mass minimally displaced posteriorly (Figure 1). The patient was given procedural sedation with Ketamine and reduced with manual in-line traction and rotation of the head back to midline in the emergency department. Post reduction CT scan showed successful correction of the C1-C2 rotatory subluxation with return of normal alignment (Figure 2). He was placed in a Miami $\mathrm{J}$ collar and discharged home from the emergency department in stable neurologic condition. Two months later, the patient returned to neurosurgery clinic with resolution of his neck pain, full range of motion, and no neurologic complaints. The hard cervical collar was removed and the patient was instructed to follow up as needed.

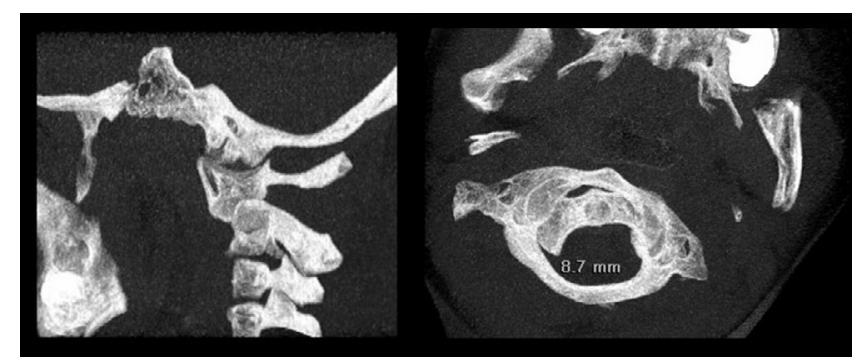

Figure 1: High resolution CT demonstrating the rotational subluxation of $\mathrm{C} 1$ to $\mathrm{C} 2$ prior to reduction. Left: Oblique view showing the subluxation of the the C1 lateral mass upon C2. Right: Axial view showing the anterior rotation and displacement of right $\mathrm{C} 1$ lateral mass upon $\mathrm{C} 2$.

${ }^{*}$ Corresponding author: Merritt D. Kinon, MD, Montefiore Medical Center/ Albert Einstein College of Medicine, Department of Neurosurgery, 3316 Rochambeau Ave, Bronx, NY 10467, USA Tel: (718) 920-7400; Fax: (718) 584-0224; E-mail: mdkinon@gmail.com

Received October 02, 2015; Accepted November 20, 2015; Published November 23, 2015

Citation: Kinon MD, Nasser R, Nakhla JP, Yassari R, Desai R, et al. (2015) Traumatic Pediatric Atlantoaxial Rotatory Subluxation Successfully Reduced and Discharged from the Emergency Department: A Case Report. J Spine S6: 005. doi:10.4172/2165-7939.S6-005

Copyright: $\odot 2015$ Merritt K, et al. This is an open-access article distributed under the terms of the Creative Commons Attribution License, which permits unrestricted use, distribution, and reproduction in any medium, provided the original author and source are credited. 


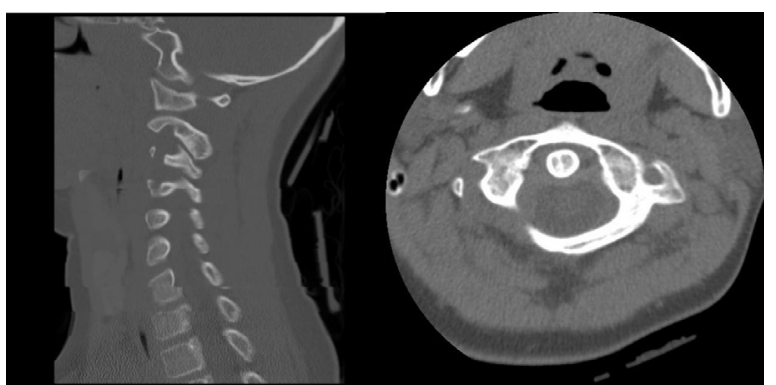

Figure 2: Post reduction cervical CT. Left: Sagittal view showing normal alignment of the $\mathrm{C} 1$ lateral mass in relation to $\mathrm{C} 2$. Right: Axial view showing resolution of the rotatory subluxation and restoration of normal alignment.

\section{Discussion}

Although atlantoaxial rotatory subluxation is uncommon, it has been described in the literature for over 100 years [10]. Fielding and Hawkings described 4 types of rotary subluxation based on the degree of displacement of the dens relative to the anterior facet of the atlas [11]. Type I is the most common and is associated with less than $3 \mathrm{~mm}$ of displacement. Type II includes displacement of 3-5 mm, and rotation in excess of that normally achieved by the C1-C2 articulation. Type III is defined by greater than $5 \mathrm{~mm}$ of displacement with weakness of the transverse and secondary ligamentous structures. Type IV, while rare, has been described mostly in adult patients with a history of rheumatoid arthritis and is related to posterior displacement of the lateral masses of $\mathrm{C} 1$ in relation to the dens $[1,11]$

Patients with torticollis associated with atlantoaxial rotatory subluxation typically assume the "cock-robin" position. It is characterized by lateral flexion of the head and neck to one side and rotation of the chin towards the opposite side $[8,11]$. Rarely is it associated with permanent neurologic deficit $[8,12]$. Patients can complain of unilateral occipital pain from compression of the second cervical nerve, since a portion of this nerve runs in the capsule of the atlantoaxial joint. The patient is apprehensive and resistant to any movement of their head because both active and passive movement is associated with severe pain. Torticollis caused by atlantoaxial subluxation can be differentiated from muscular torticollis clinically. On examination there will be a palpable deviation of the spinous process of $\mathrm{C} 2$ in the same direction as the rotation of the head, palpation of the sternocleidomastoid muscle opposite to head rotation will reveal spasm in an attempt to try to reduce the deformity, and the patient will be unable to rotate the head past midline in the direction opposite to that of the injury $[8,13]$.

The atlantoaxial joint functions primarily in rotation with excess rotation limited by the alar ligaments. Flexion and extension is limited by the transverse ligament [2]. Pediatric cases of atlantoaxial subluxation are more common than adult cases [14-16]. This may be in part due to a difference in articulation between $\mathrm{C} 1$ and $\mathrm{C} 2$ [15]. Children have unique anatomical features such as an inherent ligamentous laxity that may lead to impingement of the synovial folds at the lateral aspect of the atlantoaxial joint causing them to be more vulnerable to rotatory fixation [5,17]. Also, their facet joints are shallower and more horizontally oriented which allows greater freedom of motion, in turn making them prone to subluxations of the atlantoaxial joint and subaxial cervical spine [8].

Albeit the majority of pediatric patients presenting to the emergency department with torticollis will have a negative work up. The symptoms and deformity are usually self-limited and will resolve on their own with reassurance and symptomatic management, however, clinicians must have a high index of suspicion for atlantoaxial injury in these patients [3]. Misdiagnosis and delay in treatment can lead to a fixed, painful, and progressive cervical deformity with concomitant sequelae resulting in changes to vocal character from chronic pharyngeal compression, difficulty in opening the mouth, and abnormal posture related to elevation of one shoulder and changes in the subaxial spine $[8,11]$ Timing of treatment and degree of deformity are important in regards to prognosis [8]. Subach et al. found that patients who sought treatment 3 weeks or longer after initial injury had a higher incidence of failing nonsurgical management [8]. Pang and Li looked at both the rotational dynamics of the C1-C2 joint as well as the timing of treatment and found that delay in reducing the deformity and the greater the degree of motion abnormality of the $\mathrm{C} 1-\mathrm{C} 2$ joint correlated with a worse outcome and will likely require surgical stabilization [18]. Our patient sought treatment quickly and his atlantoaxial rotatory subluxation was a type II which is moderate in severity. Both are associated with a greater chance of treatment success with nonsurgical management alone.

It is unclear in the literature if these patients need to be admitted to the inpatient setting for treatment. Management of atlantoaxial rotatory subluxation in the pediatric population differs among treating surgeons [15]. There should be special caution regarding closed reduction of patients with AARS in the setting of trauma, or if there is any evidence of neurological deficit. Some authors recommend closed reduction followed by a course of cervical traction and prolonged hard cervical bracing while others recommend closed reduction and immobilization in a cervical orthosis reserving traction and more invasive immobilization for recurrence or treatment failures. Cervical traction is performed with the patient supine using manual hand traction at the level of the mandible or Gardner-Wells or $U$ shaped tongs (Figure 3). If tongs are utilized, they should be placed three finger breadths above the pinna, and are finger tightened. Prior tong placement, the patient must receive adequate muscle relaxants as well as analgesics. While the patient is supine, gentle traction is manually provided, with the tongs parallel to the patient's body. If the patient's neck does not return to the neutral position with manual reduction, then a 2 pound weight maybe initially added to the pulley attached to the tongs. The vector of force must be maintained so the rope and the tongs are parallel to the patient. Neurological checks should be performed after any cervical manipulation. Moreover, $\mathrm{X}$-rays should be performed with any incremental increase in weights. After satisfactory reduction has been performed, the patient may be placed in a rigid orthosis, such as a hard cervical collar. Duration of cervical immobilization also differs among authors, with most recommending between 6 weeks and 3 months of cervical immobilization after reduction. This has been deemed sufficient time to allow the ligamentous structures to heal. Surgery is reserved for treatment failures, noncompliant patients, and progressive neurologic deficits $[1,8,12,18]$. Finally, once the child is

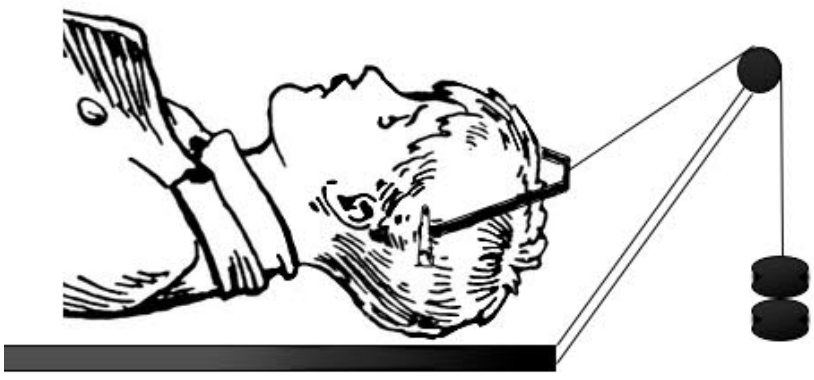

Figure 3: Illustration demonstrating reduction of atlantoaxial rotatory subluxation using traction. 
Citation: Kinon MD, Nasser R, Nakhla JP, Yassari R, Desai R, et al. (2015) Traumatic Pediatric Atlantoaxial Rotatory Subluxation Successfully Reduced and Discharged from the Emergency Department: A Case Report. J Spine S6: 005.doi:10.4172/2165-7939.S6-005

Page 3 of 3

successfully reduced back into normal alignment, it is unclear in the literature how long these pediatric patients need to be observed in the hospital and what constitutes outpatient discharge criteria [19-21].

Minor trauma often times is enough to destabilize the atlantoaxial joint, especially in children [15]. The clinician needs to have a high index of suspicion in a pediatric patient who presents with torticollis and a history of a relatively benign neck trauma. Here we have presented a case of atlantoaxial rotary subluxation after the minor trauma of a forceful throw of ball in a 9 year old patient. Swift recognition and treatment of atlantoaxial rotatory subluxation in children has been associated with lower rates of treatment failure and a good chance of complete resolution with nonoperative strategies. Since there is no clear agreed upon treatment protocol in the literature, we have shown that atlantoaxial rotatory subluxation can be successfully managed in the emergency department by manual reduction under minimal procedural sedation and once successfully reduced, if the patient and family are comfortable and motivated, they can be discharged home without the need for a prolonged hospital stay and followed as an outpatient with good clinical result.

\section{References}

1. Lee SC, Lui TN, Lee ST (2002) Atlantoaxial rotatory subluxation in skeletally immature patients. Br J Neurosurg 16: 154-157.

2. Muñiz AE, Belfer RA (1999) Atlantoaxial rotary subluxation in children. Pediatr Emerg Care 15: 25-29.

3. Sobolewski BA, Mittiga MR, Reed JL (2008) Atlantoaxial rotary subluxation after minor trauma. Pediatr Emerg Care 24: 852-856.

4. Krishnan P, Kartikueyan $\mathrm{R}$ (2015) Traumatic atlantoaxial rotatory subluxation with remote cervical spinal cord contusion in a child. Neurol India 63: 279-281.

5. Crook TB, Eynon CA (2005) Traumatic atlantoaxial rotatory subluxation. Emerg Med J 22: 671-672.

6. El-Khoury GY, Clark CR, Gravett AW (1984) Acute traumatic rotatory atlantoaxial dislocation in children. A report of three cases. The Journal of bone and joint surgery. American volume 66: 774-777.

7. Hicazi A, Acaroglu E, Alanay A, Yazici M, Surat A (2002) Atlantoaxial rotatory fixation-subluxation revisited: a computed tomographic analysis of acute torticollis in pediatric patients. Spine 27: 2771-2715.

8. Subach BR, McLaughlin MR, Albright AL, Pollack IF (1998) Current management of pediatric atlantoaxial rotatory subluxation. Spine (Phila Pa 1976) 23: 2174-2179.

9. Miyamoto H, Doita M, Nishida K, Yagi M, Iwasaki Y, et al. (2004) Traumatic anterior atlantoaxial subluxation occurring in a professional rugby athlete: case report and review of literature related to atlantoaxial injuries in sports activities. Spine (Phila Pa 1976) 29: E61-64.

10. Corner EM (1907) Rotary dislocations of the atlas. Ann Surg 45: 9-26.

11. Fielding JW, Hawkins RJ (1977) Atlanto-axial rotatory fixation. (Fixed rotatory subluxation of the atlanto-axial joint). J Bone Joint Surg Am 59: 37-44.

12. Phillips WA, Hensinger RN (1989) The management of rotatory atlanto-axial subluxation in children. J Bone Joint Surg Am 71: 664-668.

13. Van Holsbeeck EM, MacKay NN (1989) Diagnosis of acute atlanto-axial rotatory fixation. J Bone Joint Surg $\mathrm{Br} 71$ : 90-91.

14. McGrory BJ, Klassen RA, Chao EY, Staeheli JW, Weaver AL (1993) Acute fractures and dislocations of the cervical spine in children and adolescents. $J$ Bone Joint Surg Am 75: 988-995.

15. Lustrin ES, Karakas SP, Ortiz AO, Cinnamon J, Castillo M, et al. (2003) Pediatric cervical spine: normal anatomy, variants, and trauma. Radiographics 23: $539-560$.

16. Roche C, Carty H (2001) Spinal trauma in children. Pediatr Radiol 31: 677-700.

17. Kawabe N, Hirotani H, Tanaka O (1989) Pathomechanism of atlantoaxial rotatory fixation in children. J Pediatr Orthop 9: 569-574.

18. Pang D, Li V (2005) Atlantoaxial rotatory fixation: part 3-a prospective study of the clinical manifestation, diagnosis, management, and outcome of children with alantoaxial rotatory fixation. Neurosurgery 57: 954-972.

19. Phillips WA, Hensinger RN (1989) The management of rotatory atlanto-axial subluxation in children. J Bone Joint Surg Am 71: 664-668.

20. Münch C, Linhart W, Storck A, Papavero L, Windolf J, et al. (2005) Treatment of traumatic rotatory atlanto-axial subluxation in childhood. Case report and literature review. Unfallchirurg 108: 987-990.

21. de Beer JD, Hoffman EB, Kieck CF (1990) Traumatic atlantoaxial subluxation in children. J Pediatr Orthop 10: 397-400. 